\title{
Experimental Separation of Subcycle Ionization Bursts in Strong-Field Double Ionization of $\mathrm{H}_{2}$
}

\author{
Václav Hanus $\odot,{ }^{1, *}$ Sarayoo Kangaparambil, ${ }^{1}$ Seyedreza Larimian, ${ }^{1}$ Martin Dorner-Kirchner $\odot,{ }^{1}$ Xinhua Xie (谢新华)๑, ${ }^{1,2}$ \\ Markus S. Schöffler, ${ }^{3}$ Gerhard G. Paulus, ${ }^{4}$ Andrius Baltuška, ${ }^{1}$ André Staudte, ${ }^{5}$ and Markus Kitzler-Zeiler(i) ${ }^{1,}$ \\ ${ }^{1}$ Photonics Institute, Technische Universität Wien, A-1040 Vienna, Austria \\ ${ }^{2}$ SwissFEL, Paul Scherrer Institute, 5232 Villigen PSI, Switzerland \\ ${ }^{3}$ Institut für Kernphysik, Goethe-Universität, D-60438 Frankfurt am Main, Germany \\ ${ }^{4}$ Institute for Optics and Quantum Electronics, Friedrich-Schiller-Universität Jena, D-07743 Jena, Germany \\ ${ }^{5}$ Joint Attosecond Science Lab of the National Research Council and the University of Ottawa, Ottawa, Ontario K1A OR6, Canada
}

(Received 18 September 2019; accepted 10 February 2020; published 12 March 2020)

\begin{abstract}
We report on the unambiguous observation of the subcycle ionization bursts in sequential strong-field double ionization of $\mathrm{H}_{2}$ and their disentanglement in molecular frame photoelectron angular distributions. This observation was made possible by the use of few-cycle laser pulses with a known carrier-envelope phase, in combination with multiparticle coincidence momentum imaging. The approach demonstrated here will allow sampling of the intramolecular electron dynamics and the investigation of charge-statespecific Coulomb distortions on emitted electrons in polyatomic molecules.
\end{abstract}

DOI: $10.1103 /$ PhysRevLett.124.103201

The creation of free or bound electron wave packets with a sub-laser-cycle temporal structure using intense ultrashort laser pulses is at the heart of most processes in attosecond physics [1-4]. The strong oscillating electric field of the laser light can cause ionization of a system via half-cycle periodic emissions of electron wave packets and imprints a dominant subcycle temporal structure onto them. In addition, these periodically emitted wave packets, known as subcycle ionization bursts, may be modulated by the nonadiabatic response of the strongly driven system [4-7]. In strong-field double ionization, the subcycle ionization bursts from the first ionization step can be correlated with the ionization bursts from the second ionization step. Since each ionization burst corresponds to a specific time within the laser pulse envelope, various delays between the two ionization steps are sampled within a single pulse. In molecules, the delay between the two ionization steps can determine the outcome of molecular fragmentation reactions [8,9], constitutes the probe delay for intramolecular electron dynamics [5,7,10], and determines the molecular site from where the photoelectron originates [11-13]. To extract the ultrafast dynamics underlying these processes from experiments, it is necessary to disentangle the separate contributions of the subcycle ionization bursts in measured photoelectron or photoion distributions. However, so far, such disentanglement has been achieved only for atomic systems in both the sequential [14] and nonsequential [15] regimes of double ionization.

In this Letter, we demonstrate a method that allows for the unambiguous identification and extraction of the contributions from specific subcycle ionization bursts in electron momentum distributions measured for molecular double ionization. Using the example of the $\mathrm{H}_{2}$ molecule, we show that we can measure the molecular frame photoelectron angular distributions (MF-PADs) for a specific pair of two time-ordered subcycle ionization bursts in the sequential double ionization (SDI) process $\mathrm{H}_{2} \rightarrow$ $\mathrm{H}_{2}^{+}+e^{-} \rightarrow \mathrm{H}^{+}+\mathrm{H}^{+}+2 e^{-}$. The observation of the subcycle bursts and the unambiguous assignment of the two detected electrons to the first and second ionization burst was accomplished by combining our technique developed for atoms [14] with our recently published method for the reconstruction of molecular dynamics during laser interaction [16].

In our experiments, we used a reaction microscope [17] to measure in coincidence the momenta of two protons and two electrons created upon interaction of a cold jet of $\mathrm{H}_{2}$ with intense, elliptically polarized few-cycle laser pulses. The ellipticity, defined as the ratio of the electric field strength perpendicular and parallel to the main axis of the polarization ellipse, was $E_{\perp} / E_{\|}=0.85$, cf. Fig. 1(a). The laser center wavelength was $\lambda=750 \mathrm{~nm}$. Thus, the laser oscillation period $T=2 \pi / \omega=2.5$ fs, with $\omega=2 \pi c / \lambda$ as the frequency and $c$ as the speed of light. The duration of the pulses $(\approx 4.5 \mathrm{fs})$ and their carrier-envelope phases (CEPs) were measured with a stereoelectron spectrometer in phase-tagging mode $[18,19]$. The laser beam was focused in an ultrahigh vacuum chamber (base pressure $10^{-10}$ mbar) onto a supersonic gas jet of $\mathrm{H}_{2}$. Ions and electrons emerging from the interaction volume were guided by weak electric $(21 \mathrm{~V} / \mathrm{cm})$ and magnetic fields $(12 \mathrm{G})$ to two position-sensitive detectors. The momentum of the second electron was calculated from the momenta 

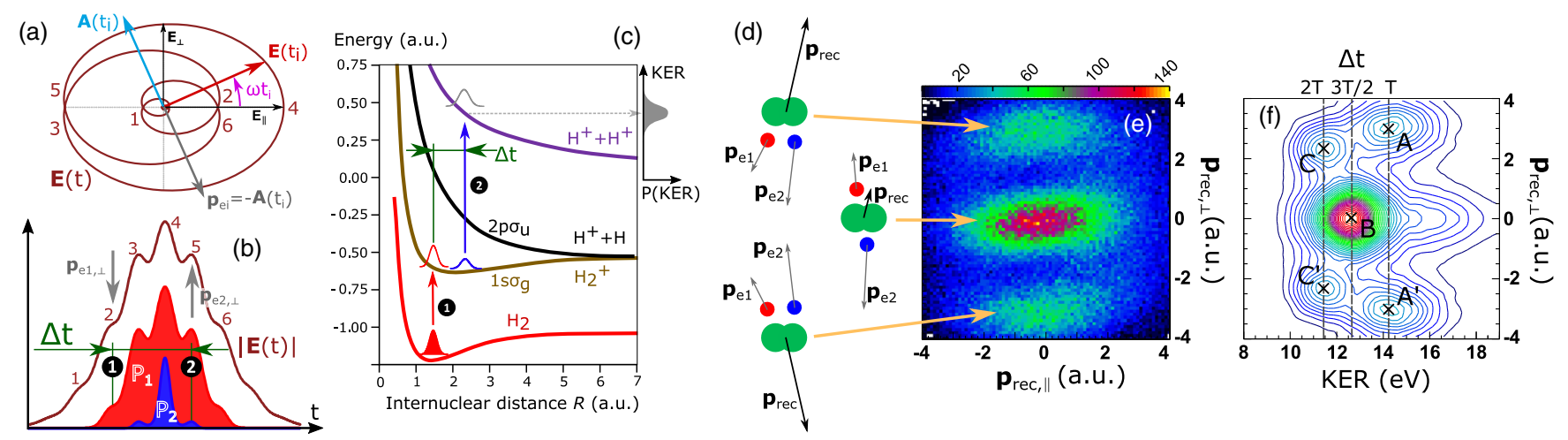

FIG. 1. (a) Electric field $\mathbf{E}(t)$ in the polarization plane of a pulse with CEP $=0$. The electric field $\mathbf{E}\left(t_{i}\right)$ and vector potential $\mathbf{A}\left(t_{i}\right)$ at the instant of the emission $t_{i}$ of an electron with momentum $\mathbf{p}_{e i}$ are indicated. (b) Absolute value of the field evolution shown in (a), $|\mathbf{E}(t)|$. The red and blue areas, denoted $P_{1}$ and $P_{2}$, sketch the single and double ionization rates, respectively, with the subcycle ionization bursts at the peaks of $|\mathbf{E}(t)|$ (numbered 1-6). Two ionization instants delayed by $\Delta t=1.5 T$ with each other, marked by 1 and $\mathbf{2}$, and the corresponding dominant directions of the $\perp$ momenta of the two emitted electrons $\mathbf{p}_{e 1, \perp}$ and $\mathbf{p}_{e 2, \perp}$ are indicated. (c) Potential energy curves of $\mathrm{H}_{2}$ relevant for SDI with a delay $\Delta t$ between the two ionization steps (1) and (2) (d) Sketches of momentum vectors for three cases of SDI dynamics corresponding to the three lobes in (e), as indicated by arrows. (e) Measured recoil momentum vector $\mathbf{p}_{\text {rec }}=\mathbf{p}_{\mathrm{H}^{+}}+\mathbf{p}_{\mathrm{H}^{+}}=-\left(\mathbf{p}_{e 1}+\mathbf{p}_{e 2}\right)$ in the laser polarization plane perpendicular $\perp$ and parallel $\|$ to the main axis of the polarization ellipse, cf. (a). (f) $\mathbf{p}_{\text {rec }, \perp}$ vs KER. The upper and lower lobes from (e) appear as separated peaks $\mathbf{A} / \mathbf{C}$ and $\mathbf{A}^{\prime} / \mathbf{C}^{\prime}$, respectively, and the center lobe appears as peak $\mathbf{B}$.

of the measured electron and both ions by exploiting momentum conservation. The laser peak intensity in situ was $9 \times 10^{14} \mathrm{~W} \mathrm{~cm}^{-2}$ [20]. Further details on the reaction microscope can be found in Refs. [21-23] and on the optical setup in Ref. [14].

When $\mathrm{H}_{2}$ undergoes SDI in a strong elliptically polarized laser field, the first ionization step at time $t_{1}$ triggers vibronic dynamics in the molecular cation $\mathrm{H}_{2}^{+}$, and the second ionization step at time $t_{2}=t_{1}+\Delta t$ initiates Coulomb explosion into $\mathrm{H}^{+}+\mathrm{H}^{+}$, cf. the sketches in Figs. 1(b) and 1(c). While the kinetic energy released, $\mathrm{KER}=1 / R$, with $R$ as the internuclear distance at which the Coulomb explosion is initiated, provides a precise measure for the nuclear motion in between the two ionization steps $[16,24,25]$, the momenta of the two emitted electrons $\mathbf{p}_{e 1, e 2}$ provide information on the emission times of the two electrons within a laser cycle. This is because in elliptically polarized light the ionization phase within a laser cycle $\omega t_{i}$ is approximately mapped onto the emission angle of the photoelectron by the laser field via $\mathbf{p}_{e i}=$ $-\mathbf{A}\left(t_{i}\right), i=\{1,2\}$ [26,27], cf. Fig. 1(a). In detail, the influence of the ion's Coulomb potential on $\mathbf{p}_{e i}$ must be considered $[7,28,29]$. The laser vector potential $\mathbf{A}(t)$ is connected to the laser electric field by $\mathbf{A}(t)=$ $-\int_{-\infty}^{t} \mathbf{E}\left(t^{\prime}\right) d t^{\prime}$. Thus, measurement of the electron emission angle in the laboratory frame determines the ionization time $t_{i}$ within one cycle $[14,16,30,31]$.

In the case of SDI, the momenta of the two emitted electrons are reflected in the recoil momentum vector of the two protons, $\mathbf{p}_{\mathrm{rec}}=\mathbf{p}_{\mathrm{H}^{+}}+\mathbf{p}_{\mathrm{H}^{+}}=-\left(\mathbf{p}_{e 1}+\mathbf{p}_{e 2}\right)$ [14,30,32]. Figure 1(e) shows the measured distribution of $\mathbf{p}_{\text {rec }}$, obtained from the ions' momenta, in the polarization plane, integrated over all values of the CEP and KER. Note, that this distribution and all other information extracted from $\mathbf{p}_{\text {rec }}$ below can also be obtained from $\mathbf{p}_{e}=\mathbf{p}_{e 1}+\mathbf{p}_{e 2}$, which makes our approach suitable also for larger molecules. In elliptical light, ionization takes place preferentially around the times when the field vector $\mathbf{E}(t)$ passes the major axis of the polarization ellipse during its rotation, i.e., twice during the optical cycle. Depending on the ionization delay $\Delta t$, the two electrons can be streaked by the laser field into the same or into opposite hemispheres. If the two electrons are ejected with a delay of an even number of half cycles $[\Delta t=2 n(T / 2) ; n=0,1,2, \ldots]$, the electrons are emitted into the same hemisphere and their momenta add up to a large value of $\mathbf{p}_{\text {rec }}$. Examples of electron momentum vectors consistent with such SDI dynamics are indicated as the upper and lower sketches in Fig. 1(d). In contrast, if the two electrons are emitted with an odd number of half cycles $[\Delta t=(2 n+1)(T / 2), n=0,1,2, \ldots]$, they are streaked into the opposite hemisphere and their momenta cancel to a small value of $\mathbf{p}_{\text {rec }}$, see the center sketch in Fig. 1(d).

The three lobes in the momentum distribution in Fig. 1(e) are, thus, the signatures of the subcycle ionization bursts emitted during various combinations of half-cycle peaks of $|\mathbf{E}(t)|$, numbered 1-6 in Fig. 1(b), integrated over the whole pulse duration and over all values of the CEP and KER. Because the ionization delay $\Delta t$ is mapped onto KER, cf. Fig. 1(c), depending on their ionization delay $\Delta t=n(T / 2), n=0,1,2, \ldots$, a certain pair of subcycle bursts leads to a certain value of KER. The three lobes in the momentum distribution Fig. 1(e) thus appear as separated peaks $\mathbf{A} / \mathbf{A}^{\prime}, \mathbf{B}$, and $\mathbf{C} / \mathbf{C}^{\prime}$ in the KER vs $\mathbf{p}_{\text {rec }, \perp}$ distribution shown in Fig. 1(f). Hence, selection of a certain 


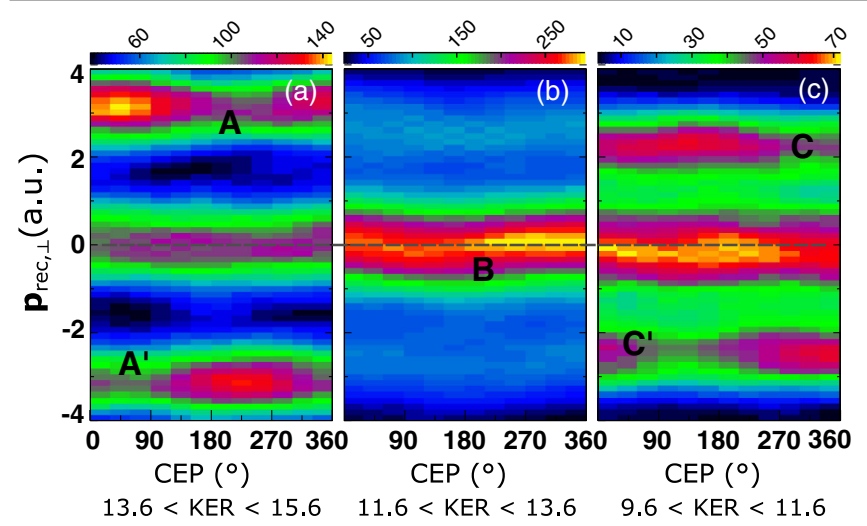

FIG. 2. Measured distributions of the recoil momentum vector along the minor polarization axis $\mathbf{p}_{\text {rec, } \perp}$ over CEP for the three indicated ranges of KER (in $\mathrm{eV}$ ): (a) $13.6<\mathrm{KER}<15.6$, (b) $11.6<\mathrm{KER}<13.6$, and (c) $9.6<\mathrm{KER}<11.6$.

range in KER allows selecting a well-defined range of $\Delta t$ values [16].

To understand how the peaks $\mathbf{A} / \mathbf{A}^{\prime}, \mathbf{B}$, and $\mathbf{C} / \mathbf{C}^{\prime}$ can be disentangled into the different contributions of specific SDI bursts, we first resolve them as a function of CEP for three different ranges of KER, each $2 \mathrm{eV}$ wide, corresponding to about $0.5 T$-wide ranges of $\Delta t$ around $1 T, 1.5 T$, and $2 T$, see Figs. 2(a)-2(c). A pronounced dependence on the CEP of both the momentum and yield is clearly visible for all peaks. Particularly strong yield modulations are observed for peaks $\mathbf{A} / \mathbf{A}^{\prime}$ and $\mathbf{C} / \mathbf{C}^{\prime}$, where for certain values of the CEP the two electrons are predominantly emitted into one hemisphere only. Additional analysis of this asymmetric two-electron emission and its application to the calibration of the CEP in the experimental distributions is provided in the Supplemental Material [33].

A qualitative understanding of the CEP dependence of the SDI dynamics can be obtained with the help of semiclassical simulations. The numerical model underlying these simulations is described in the Supplemental Material [33], and results are shown in Fig. 3. First, we apply our model to the measured momentum distributions for the highest KER range, shown in Fig. 2(a), for which $\Delta t \approx 1 T$. For this ionization delay, the two electrons are both streaked into the same hemisphere, either both upward or both downward, depending on the specific combination of subcycle bursts. The strong yield asymmetry and the dominance of the lower peak $\mathbf{A}^{\prime}$ in Fig. 2(a) for CEP = $205^{\circ}$ shows, because of $\mathbf{p}_{\mathrm{rec}, \perp}=-\left(\mathbf{p}_{e 1, \perp}+\mathbf{p}_{e 2, \perp}\right)$, that for this CEP value the two electrons are predominantly emitted only into the upward hemisphere. For CEP $=25^{\circ}$ $\left(=205^{\circ}-180^{\circ}\right)$ the opposite emission scenario and the dominance of peak $\mathbf{A}$ is observed. This implies that for these CEP values the two ionization steps each take place during predominantly only one subcycle burst: up bursts for $\mathrm{CEP}=205^{\circ}$ and down bursts for $=25^{\circ}$.

The simulated data for CEP $=205^{\circ}$ shown in the leftmost column of Fig. 3 confirm this single-burst ionization

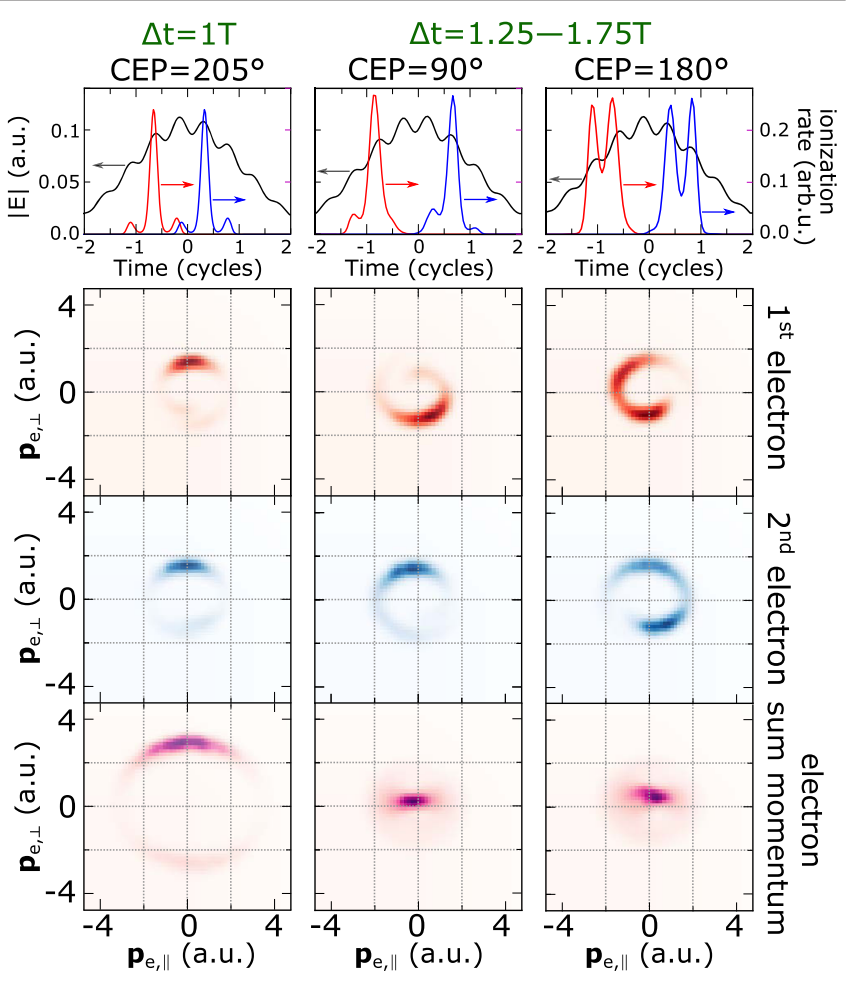

FIG. 3. (Top) Absolute value of electric field $|\mathbf{E}(t)|$ (black) and simulated SDI rates (red, blue) over time for different values of the CEP and $\Delta t$ (columns). (Bottom) Corresponding simulated electron momentum distributions in the polarization plane. See text for details.

dynamics and the experimentally observed yield asymmetry. For intermediate values of the CEP, e.g., for $\mathrm{CEP} \approx 110^{\circ}$, the numerical model reveals that more than one pair of subcycle bursts contribute and both up and down pairs are emitted. Accordingly, the measured electron yield in Fig. 2(a) does not show any asymmetry for CEP $\approx$ $110^{\circ}$ and peaks $\mathbf{A}$ and $\mathbf{A}^{\prime}$ are equally strong. The twoelectron emission dynamics for $\Delta t=2 T$ that results in the peaks $\mathbf{C} / \mathbf{C}^{\prime}$ takes place analogously to the case $\Delta t=1 T$. The measured CEP dependence of the corresponding yield distributions in Fig. 2(c) can therefore be explained qualitatively using the same argumentation.

Of particular interest is the case $\Delta t=1.5 T$. For this value, the two electrons are streaked into opposite hemispheres and dominantly only peak $\mathbf{B}$ is observed. The corresponding CEP-resolved momentum distribution in Fig. 2(b) for $\Delta t=1.5 T( \pm 0.25 T)$ shows that the mean value of peak $\mathbf{B}$ oscillates with CEP. To explain this CEP oscillation, we turn to the simulated distributions in Fig. 3. The center column shows that for $\mathrm{CEP} \approx 90^{\circ}$ dominantly only one pair of subcycle bursts is emitted symmetrically around the pulse peak. One electron is streaked upward, the other one downward, and their momenta almost cancel, resulting in very small sum momentum close to zero. For $\mathrm{CEP} \approx 180^{\circ}$ (Fig. 3, rightmost column), in contrast, each electron is emitted during two subcycle peaks and streaked 
both up- and downward. Hence, the photoelectrons are no longer emitted symmetrically around the pulse maximum, explaining the CEP oscillation of $\mathbf{p}_{\text {rec }, \perp}$. We conclude from this analysis that it is possible to control the SDI process with the CEP such that the two emitted electrons can be disentangled in the experimental distributions, i.e., that a situation as in the center column of Fig. 3 can be achieved.

The value of the CEP for which this is possible can be determined directly from experimental data without the need to consult simulations. To demonstrate this, we consult the electron energy distribution over CEP shown in Fig. 4(a). The mean energy of the electrons emitted toward the detector, marked by a red line, shows a clear oscillation with the CEP. If those electrons that are streaked into the opposite hemisphere (away from the detector) are selected, an oscillation phase shifted by $180^{\circ}$ is obtained (blue line). From the discussions above, we know that if each electron is emitted during one subcycle burst only, the two electrons will be emitted symmetrically around the pulse peak-one upward, the other downward. As a consequence, both electrons must have very similar energy. Thus, for symmetry reasons, the CEP at which this situation occurs is that where the energy of the up- and downward electrons is the same. This is, in accordance with the simulations, the case for $\mathrm{CEP}=90^{\circ}$ (and $\mathrm{CEP}=270^{\circ}$ ), indicated by a black circle in Fig. 4(a). The corresponding

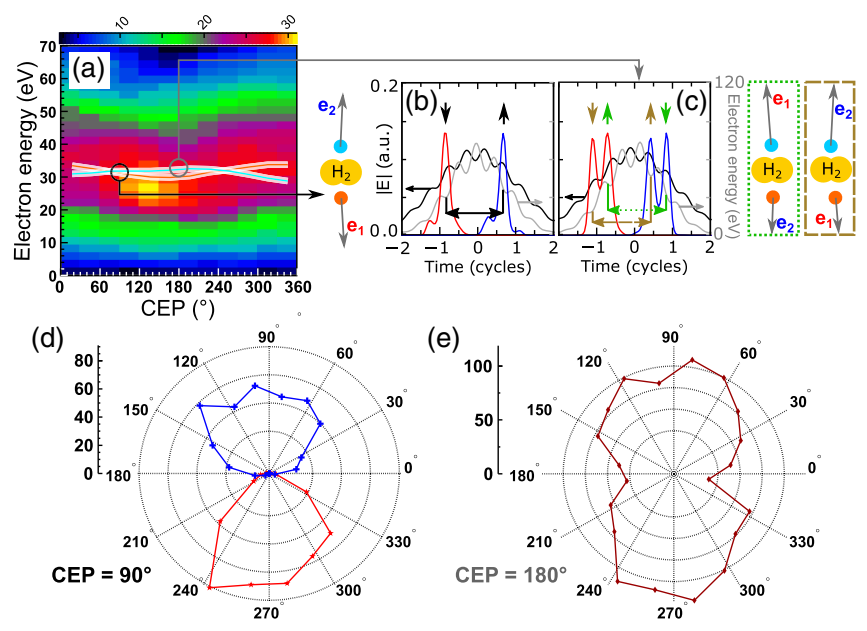

FIG. 4. (a) Measured energy distribution of electrons for $\Delta t=1.5 T( \pm 0.25 T) \quad(11.6<\mathrm{KER}<13.6 \mathrm{eV}$, peak B $)$ over CEP emitted into the hemisphere facing the detector. The average energy is shown by a red line. The blue line denotes the average energy emitted into the opposite hemisphere. (b), (c) Simulated subcycle emission bursts (reproduced from Fig. 3) for the same range of $\Delta t$ as in (a) for two values of the CEP indicated by circles and arrows. The gray lines depict the electrons' energy $\propto|A(t)|^{2}$. The cartoons left [right] of (b) [(c)] visualize the corresponding momentum vectors of the two emitted electrons. The two possible cases in (c) are indicated by brown and green colors, respectively. (d) MF-PAD corresponding to (b). (e) MF-PAD corresponding to (c). emission scenario and the simulated subcycle bursts in the time domain (reproduced from Fig. 3) are sketched in Fig. 4(b). On the contrary, when the electrons are emitted during more than one pair of subcycle bursts, the up- and downward electrons will have markedly different energy. This is the case for CEP $=180^{\circ}$, indicated by a gray circle in Fig. 4(a); the corresponding emission scenario is sketched in Fig. 4(c).

Figure 4 thus holds experimental proof that for $\mathrm{CEP} \approx 90^{\circ}$ and $\mathrm{CEP} \approx 270^{\circ}$ it becomes possible to disentangle the contributions of the subcycle bursts in electron momentum distributions simply by selecting one electron from the upward and the other from the downward hemisphere. Moreover, based on the known rotation direction of $\mathbf{E}(t)$ determined by the helicity of the laser field, it is even feasible to determine during which of the two subcycle bursts a specific electron has been emitted. For the helicity used in the experiment and for CEP $=90^{\circ}$, the first emitted electron is streaked downward [cf. Fig. 4(b)], and for $\mathrm{CEP}=270^{\circ}$, the first emitted electron is streaked upward. With that, by fixing the CEP to one of the two values, the SDI dynamics is completely determined.

This opens up the possibility to obtain an experimental trace of the attosecond evolution of the subcycle ionization bursts. By exploiting the fact that the electron emission dynamics is streaked into the angular direction by the rotating laser field, we can represent the attosecond evolution of the subcycle two-electron bursts as photoelectron angular distributions. Moreover, because in our experiment we also measure the orientation of the molecules in the lab frame via the momentum vectors of the two protons, we are able to plot the PADs in the molecular frame of reference. The MF-PADs for CEP $=90^{\circ}$ and $\mathrm{CEP}=180^{\circ}$ are shown in Figs. 4(d) and 4(e). Because for $\mathrm{CEP}=180^{\circ}$ two pairs of subcycle bursts are emitted, the MF-PAD cannot be separated without ambiguity, cf. Fig. 4(e). In contrast, the dominant single pair emitted for CEP $=90^{\circ}$ can be unambiguously disentangled in the MF-PAD, see Fig. 4(d).

The capability of obtaining absolutely defined traces of the attosecond two-electron emission for specific molecular orientations in the laser polarization plane opens up intriguing possibilities. For example, it allows us to trace the intramolecular electron dynamics in between the two ionization steps $[5,6,10]$. These dynamics can lead to a very complicated pattern of the intramolecular charge density $[7,10]$, which may modulate the ionization rate of the second ionization step on subcycle times. As the subcycle dynamics of the second ionization step is reflected in the angular intensity distribution of the corresponding lobe in the MF-PAD that can be unambiguously extracted from the electron momentum data using our approach, these modulations could be extracted using suitable models. Based on the possibility that the electron momentum distribution can be unambiguously separated into the contributions from the 
first and second electron emission, our method can also be applied to determining the Coulomb distortion [28,29] imparted by the singly, respectively, doubly charged parent ion onto the first, respectively, second emitted electron.

In conclusion, we observed the subcycle ionization bursts in SDI of $\mathrm{H}_{2}$ with elliptically polarized intense few-cycle laser pulses with a known CEP using multiparticle coincidence momentum imaging. This permitted us to measure the MF-PAD for a single pair of subcycle bursts that leads to double ionization, and the unambiguous assignment of each burst to one of the two detected electrons. Although demonstrated for $\mathrm{H}_{2}$, we expect that the approach demonstrated here will allow the observation of the subcycle ionization bursts also in more complicated molecules, as the only requirement for the molecule is that its fragmentation dynamics is commensurate with the fewcycle laser pulse duration, which can be widely fulfilled using longer wavelength fields. This will grant experimental access to otherwise inaccessible quantities such as the Coulomb distortion for specific charge states and, particularly interesting, to intramolecular electron localization dynamics on attosecond timescales in polyatomic molecules in between the two ionization steps.

This work was financed by the Austrian Science Fund (FWF), Grants No. P28475-N27 and No. P30465-N27.

*vaclav.hanus@tuwien.ac.at †markus.kitzler-zeiler@tuwien.ac.at

[1] R. Kienberger, M. Hentschel, M. Uiberacker, C. Spielmann, M. Kitzler, A. Scrinzi, M. Wieland, T. Westerwalbesloh, U. Kleineberg, U. Heinzmann, M. Drescher, and F. Krausz, Science 297, 1144 (2002).

[2] J. Xu, Z. Chen, A.-T. Le, and C. D. Lin, Phys. Rev. A 82, 033403 (2010).

[3] M. Haertelt, X.-B. Bian, M. Spanner, A. Staudte, and P. B. Corkum, Phys. Rev. Lett. 116, 133001 (2016).

[4] X. Xie, S. Roither, D. Kartashov, E. Persson, D. G. Arbó, L. Zhang, S. Gräfe, M. S. Schöffler, J. Burgdörfer, A. Baltuška, and M. Kitzler, Phys. Rev. Lett. 108, 193004 (2012).

[5] N. Takemoto and A. Becker, Phys. Rev. Lett. 105, 203004 (2010).

[6] M. Odenweller, N. Takemoto, A. Vredenborg, K. Cole, K. Pahl, J. Titze, L. P. H. Schmidt, T. Jahnke, R. Dörner, and A. Becker, Phys. Rev. Lett. 107, 143004 (2011).

[7] M. Spanner, S. Gräfe, S. Chelkowski, D. Pavičić, M. Meckel, D. Zeidler, A. B. Bardon, B. Ulrich, A. D. Bandrauk, D. M. Villeneuve, R. Dörner, P. B. Corkum, and A. Staudte, J. Phys. B 45, 194011 (2012).

[8] X. Xie, E. Lötstedt, S. Roither, M. Schöffler, D. Kartashov, K. Midorikawa, A. Baltuška, K. Yamanouchi, and M. Kitzler, Sci. Rep. 5, 12877 (2015).

[9] P. Sándor, V. Tagliamonti, A. Zhao, T. Rozgonyi, M. Ruckenbauer, P. Marquetand, and T. Weinacht, Phys. Rev. Lett. 116, 063002 (2016).

[10] H. Hennig, J. Breidbach, and L. S. Cederbaum, J. Phys. Chem. A 109, 409 (2005).
[11] H. Ohmura, N. Saito, and M. Tachiya, Phys. Rev. Lett. 96, 173001 (2006).

[12] J. Wu, M. Meckel, L. Schmidt, M. Kunitski, S. Voss, H. Sann, H. Kim, T. Jahnke, A. Czasch, and R. Dörner, Nat. Commun. 3, 1113 (2012).

[13] K. Liu and I. Barth, Phys. Rev. Lett. 119, 243204 (2017).

[14] M. S. Schöffler, X. Xie, P. Wustelt, M. Möller, S. Roither, D. Kartashov, A. M. Sayler, A. Baltuska, G. G. Paulus, and M. Kitzler, Phys. Rev. A 93, 063421 (2016).

[15] B. Bergues, M. Kübel, N. G. Johnson, B. Fischer, N. Camus, K. J. Betsch, O. Herrwerth, A. Senftleben, A. M. Sayler, T. Rathje, T. Pfeifer, I. Ben-Itzhak, R. R. Jones, G. G. Paulus, F. Krausz, R. Moshammer, J. Ullrich, and M. F. Kling, Nat. Commun. 3, 813 (2012).

[16] V. Hanus, S. Kangaparambil, S. Larimian, M. DornerKirchner, X. Xie, M. S. Schöffler, G. G. Paulus, A. Baltuška, A. Staudte, and M. Kitzler-Zeiler, Phys. Rev. Lett. 123, 263201 (2019).

[17] R. Dörner, V. Mergel, O. Jagutzki, L. Spielberger, J. Ullrich, R. Moshammer, and H. Schmidt-Böcking, Phys. Rep. 330, 95 (2000).

[18] A. M. Sayler, T. Rathje, W. Müller, K. Rühle, R. Kienberger, and G. G. Paulus, Opt. Lett. 36, 1 (2011).

[19] T. Rathje, N. G. Johnson, M. Möller, F. Süßmann, D. Adolph, M. Kübel, R. Kienberger, M. F. Kling, G. G. Paulus, and A. M. Sayler, J. Phys. B 45, 074003 (2012).

[20] C. Smeenk, J. Z. Salvail, L. Arissian, P. B. Corkum, C. T. Hebeisen, and A. Staudte, Opt. Express 19, 9336 (2011).

[21] X. Xie, K. Doblhoff-Dier, S. Roither, M. S. Schöffler, D. Kartashov, H. Xu, T. Rathje, G. G. Paulus, A. Baltuška, S. Gräfe, and M. Kitzler, Phys. Rev. Lett. 109, 243001 (2012).

[22] L. Zhang, X. Xie, S. Roither, D. Kartashov, Y. L. Wang, C. L. Wang, M. Schöffler, D. Shafir, P. B. Corkum, A. Baltuška, I. Ivanov, A. Kheifets, X. J. Liu, A. Staudte, and M. Kitzler, Phys. Rev. A 90, 061401(R) (2014).

[23] X. Xie, T. Wang, S. G. Yu, X. Y. Lai, S. Roither, D. Kartashov, A. Baltuška, X. J. Liu, A. Staudte, and M. Kitzler, Phys. Rev. Lett. 119, 243201 (2017).

[24] H. Niikura, F. Légaré, R. Hasbani, M. Y. Ivanov, D. M. Villeneuve, and P. B. Corkum, Nature (London) 421, 826 (2003).

[25] T. Ergler, A. Rudenko, B. Feuerstein, K. Zrost, C. D. Schröter, R. Moshammer, and J. Ullrich, Phys. Rev. Lett. 97, 193001 (2006).

[26] F. Faisal, J. Phys. B 6, L89 (1973).

[27] H. Reiss, Phys. Rev. A 22, 1786 (1980).

[28] P. Eckle, A. N. Pfeiffer, C. Cirelli, A. Staudte, R. Dörner, H. G. Muller, M. Büttiker, and U. Keller, Science 322, 1525 (2008).

[29] L. Torlina, F. Morales, J. Kaushal, I. Ivanov, A. Kheifets, A. Zielinski, A. Scrinzi, H. G. Muller, S. Sukiasyan, M. Ivanov, and O. Smirnova, Nat. Phys. 11, 503 (2015).

[30] C. M. Maharjan, A. S. Alnaser, X. M. Tong, B. Ulrich, P. Ranitovic, S. Ghimire, Z. Chang, I. V. Litvinyuk, and C. Cocke, Phys. Rev. A 72, 041403(R) (2005).

[31] P. Eckle, M. Smolarski, P. Schlup, J. Biegert, A. Staudte, M. Schöffler, H. G. Muller, R. Dörner, and U. Keller, Nat. Phys. 4, 565 (2008).

[32] A. N. Pfeiffer, C. Cirelli, M. Smolarski, R. Dörner, and U. Keller, Nat. Phys. 7, 428 (2011). 
[33] See Supplemental Material at http://link.aps.org/ supplemental/10.1103/PhysRevLett.124.103201 for details of the simulations, which includes Refs. [34-36], and the CEP dependence of the two-electron emission asymmetry.
[34] X. M. Tong and C.D. Lin, Phys. Rev. A 70, 023406 (2004).

[35] V. H. Dibeler, R. M. Reese, and M. Krauss, J. Chem. Phys. 42, 2045 (1965).

[36] T. Sharp, At. Data Nucl. Data Tables 2, 119 (1970). 